\title{
School Health Nursing
}

National Cancer Institute

\section{Source}

National Cancer Institute. School Health Nursing. NCI Thesaurus. Code C19023.

Nursing care given to those in school settings 